\section{Probable association of neonatal death with the use of tramadol to treat labour pain}

\author{
ŠTEFAN GROSEK • MARTINA SOBAN • ALENKA KUŠTRIN-SAMBA \\ JANEZ PRIMOŽIČ • IZTOK GRABNAR
}

IZTOK GRABNAR ( $\square)$ Faculty of Pharmacy, University of Ljubljana Aškerčeva cesta 7, 1000 Ljubljana, Slovenia Phone: +38614769543

Fax: + 38614258031 E-mail: iztok.grabnar@ffa.uni-lj.si

ŠTEFAN GROSEK • JANEZ PRIMOŽIČ Department of Paediatric Surgery and Intensive Therapy University Medical Centre Ljubljana Bohoričeva 20, 1000 Ljubljana, Slovenia Medical Faculty, University of Ljubljana Vrazov trg 2, 1000 Ljubljana, Slovenia

MARTINA SOBAN

Community Health Centre Koper Dellavallejeva ulica 3, 6000 Koper, Slovenia

ALENKA KUŠTRIN-SAMBA Institute of Forensic Medicine Medical Faculty, University of Ljubljana Korytkova ulica 2, 1000 Ljubljana, Slovenia

\begin{abstract}
Tramadol is often used in obstetrics for the relief of labour pain. It has a dual mechanism of action, a monoaminergic effect of tramadol itself and an opioid effect, primarily mediated by its metabolite O-desmethyl tramadol, formed by genetically polymorphic cytochrome P450 2D6. In newborns, elimination of O-desmethyl tramadol is prolonged due to immature renal function. We report on a case of neonatal death following rectal administration of tramadol to treat labour pain to a birth-giving mother. An objective causality assessment using the Naranjo probability scale revealed that the likelihood of tramadol causing respiratory depression was probable. We hypothesize that neonatal death was associated with an increased exposure to O-desmethyl tramadol due to ultrarapid metabolizer cytochrome P450 2D6 genotype of the mother. More evidence is needed to support this association. Nevertheless, in obstetric analgesia, tramadol should be used with more caution until more safety data are available.
\end{abstract}

Key words: opioid analgesics, tramadol, O-desmethyltramadol, labour, drug toxicity, respiratory depression

\section{Introduction}

Tramadol is a centrally acting analgesic. In humans it is extensively metabolized, with major metabolites being O-desmethyl (M1) and N-desmethyl (M2) tramadol. Both, tramadol and its metabolite M1 are agonists of the $\mu$ opioid receptor. Compared to parent tramadol, M1 has approximately 700 fold higher affinity for the $\mu$-receptors. (1) Consequently, M1 metabolite is predominantly responsible for the $\mu$-receptor mediated analgesic effect of tramadol, although nonopioid mechanisms of action potentiate opioid effects. (2) The dual mode of action of tramadol by inhibition of noradrenaline and serotonin reuptake, besides agonism at the opioid receptors, causes decreased risk of respiratory depression. $(3,4)$ In obstetrics it is used to treat labour pain. (5) It was demonstrated that tramadol
(50-100 mg) in the first stage of labour is as effective as pethidine (50-75 mg), but has a superior safety profile. (6) In vitro it was demonstrated that formation of M1 is catalyzed by the liver enzyme cytochrome P450 (CYP) 2D6. (7) The gene encoding for CYP2D6 is polymorphic resulting in functionally different enzymes, characterized in poor (PM), intermediate (IM), extensive (EM) and ultrarapid (UM) metabolizer phenotypes. Tramadol and M1 pass the placental barrier. (8) Depending on the 
postmenstrual age, neonates possess almost complete hepatic capacity of tramadol metabolism to $\mathrm{M} 1$; however, compared to mothers, the renal elimination of $\mathrm{M} 1$ is notably prolonged. $(8,9)$ Despite its use in the treatment of labour pain for over a decade, there is no report, to our knowledge, of a fatal event with such use.

This case report suggests a probable association between fatal respiratory depression in a neonate and antenatal administration of tramadol in the mother for labour pain.

\section{Case report}

For the 28 year old female this was her third pregnancy. Four weeks before delivery (in the 36th week of gestation) she had successful external cephalic version. Finally, labour was induced with oxytocin in the 40th week, at 10.30 , because of 2 hours of decelerations on cardiotocography. At 13.50 the mother was given one suppository of $100 \mathrm{mg}$ tramadol hydrochloride (Tadol $\AA$, Krka, d.d., Novo mesto, Slovenia) and one suppository of $10 \mathrm{mg}$ hyoscine butylbromide (Buscopan ${ }^{\circledR}$, Boehringer Ingelheim International $\mathrm{GmBH}$, Ingelheim am Rhein, Germany) for labour pain.

At 15.16 a boy $(2910 \mathrm{~g})$ was born with an APGAR score of 8 and 9 at 1 and 5 minutes after birth, respectively. The baby stayed with his mother, but at 16.50 he was found pulseless and apnoeic. He was cyanotic and hypotonic. The physicians started cardiopulmonary resuscitation and $0.2 \mathrm{mg}$ of adrenaline was delivered through an endotracheal tube. After 2 minutes heart rate was re-established.

At the arrival of the transport team, the newborn was already intubated and ventilated by the attending physician. His pulse rate was over 100 per minute, blood pressure was $89 / 51 \mathrm{mmHg}$ (left arm) and 86/55 mmHg (left leg). He was floppy, not reacting to any stimuli. His pupils were constricted and not reacting to light. The newborn was transported to the Level III Neonatal and Paediatric Intensive Care Unit.

A brain ultrasound was performed by an experienced paediatric radiologist.
The ultrasound revealed diffuse hypoechogenic areas and small hyperechogenic areas periventriculary. Induced hypothermia (core temperature was held between 32 and $34{ }^{\circ} \mathrm{C}$ ) for neuroprotection and therapy with phenobarbital was started. The Electroencephalogram (EEG) was repeatedly (3 times) abnormal and showed changes in activity, which were attributed to diffuse ischaemic lesions of the white substance. A neurologist confirmed the minimal status of consciousness of the newborn and no resuscitation was indicated. The child died 28 days after birth.

Blood samples were drawn from the mother at $5 \mathrm{~h}$ and from the newborn at 7 and $72 \mathrm{~h}$ after birth. Tramadol concentration in the serum was determined using a gas chromatography-mass spectrometry (GC-MS) assay. The lower limit of quantification (LLOQ) of the method was $0.04 \mathrm{mg} / \mathrm{l}$. Tramadol serum concentration in the mother was $0.18 \mathrm{mg} / \mathrm{l}$ (therapeutic range 0.1-0.3 $\mathrm{mg} / \mathrm{l})$. In the newborn, tramadol serum concentration at $7 \mathrm{~h}$ after birth was 0.05 $\mathrm{mg} / \mathrm{l}$. A second measurement in the newborn, at $72 \mathrm{~h}$ was below the assay LLOQ.

\section{Discussion}

Labour pain inevitably affects maternal psychology, labour progress and foetal well-being. Consequently, adequate analgesia is essential in obstetrics. (10) Because of the lower incidence of side effects, and analgesic activity comparable to pethidine, tramadol has been recommended for obstetrical analgesia. $(6,11)$ Additionally, in the study by Khooshideh and Shahriari the duration of labour was shorter for the first stage (190 vs. $140 \mathrm{~min}$ ) and for the second stage (33 vs. $25 \mathrm{~min}$ ) in women treated with tramadol compared to pethidine. (11) In a previous study by Keskin et al., however, no difference with regard to the duration of labour and APGAR score was found, while superior analgesic efficacy was observed in women treated with pethidine. (10) In general, tramadol is considered to have fewer cardiovascular, gastrointestinal and central depressant adverse effects than any other opioid drug. $(2,3)$ The most common adverse effects observed with tramadol are: central nervous system (CNS) depression (27.4\%), nausea and vomiting (21.1\%), tachycardia (17.4\%), seizures (13.7\%), confusion (3.7\%) and miosis (3.2\%). (12) CNS depression can be successfully treated with naloxone and seizures with benzodiazepines. Dual mechanism of action and relatively slow metabolism of tramadol to M1 result in prolonged analgesia. Consequently, repeated doses of naloxone are necessary to antagonize the opioid adverse effects. The influence of CYP2D6 polymorphism on tramadol pharmacokinetics has been extensively studied. However, its clinical importance regarding analgesic efficacy and safety has not been elucidated, especially in patients with UM phenotype. Based on the known mechanisms of action and the identified effects of CYP2D6 genotype on drug exposure, increased incidence of serotonergic effects is expected in patients with PM phenotype, while opioid mediated adverse effects are more likely in patients with UM phenotype. Tramadol and M1 have a high placental permeability and are not lost during foetal passage. In neonates, renal elimination of $\mathrm{M} 1$ is considerably prolonged, especially in the first hours post-partum. (8) Based on data by Stamer et al., the plasma concentrations of M1 in adult patients with UM phenotype is increased by more than $100 \%$, compared to EM phenotype. (13) The exposure of the neonate to $M 1$, however, is hard to predict as it also depends on the time interval between drug administration and delivery of the child. While most people have EM phenotype, approximately $1 \%$ of people in Finland, Denmark (14) and Slovenia, (15) 2\% in Germany, (16) $3.5 \%$ in Spain, (16) $10 \%$ in Greece and Portugal, (14) and 29\% in Ethiopia (14) have CYP2D6 gene duplication resulting in UM phenotype.

The clinical signs presented in this case are consistent with opioid toxicity leading to respiratory depression and neonatal death. Furthermore, opioid induced respiratory depression may have 
been enhanced by the anticholinergic action of hyoscine butylbromide. (17) According to the Naranjo probability scale, (18) respiratory depression was probably related to the tramadol treatment $(\mathrm{Q} 1=0 ; \mathrm{Q} 2=2 ; \mathrm{Q} 3=0$; $\mathrm{Q} 4$ $=0 ; \mathrm{Q} 5=2 ; \mathrm{Q} 6=0 ; \mathrm{Q} 7=0 ; \mathrm{Q} 8=$ $0 ; \mathrm{Q9}=0 ; \mathrm{Q10}=1$, total score $=5$ ). The measured serum concentration of tramadol in the mother at $6.5 \mathrm{~h}$ after administration of tramadol suppository was within the therapeutic range and consistent with the mean observed tramadol concentration with this type of administration (approximately $0.2 \mathrm{mg} / \mathrm{l}$ ). (19) However, it is the formation of M1, which is probably related to the observed respiratory depression in the neonate. Unfortunately, M1 concentration was not available, since this metabolite is not routinely measured in our toxicology lab. Some pharmacokinetic data suggest that formation clearance of M1 is a minor route in the total elimination of tramadol. $(20,21)$ Consequently, genetic polymorphism of CYP2D6 has a more pronounced effect on the serum concentration time course of $\mathrm{M} 1$ than the concentration time course of tramadol. This is also supported by the study of tramadol pharmacokinetics in mothers treated for labour pain and their neona- tes, where the serum concentration of tramadol in neonates at the time of birth was much less variable (range $0.122-$ $0.557 \mathrm{mg} / \mathrm{l})$ then the serum concentration of M1 (range 0.003-0.112 mg/l). (8) Based on these data we speculate that in the presented case, the mother has a UM phenotype resulting in minor influence on the exposure to tramadol, but considerably increased exposure to M1. At the time of delivery, serum concentrations of $\mathrm{M} 1$ in the mother and the child were presumably near maximum. Due to immature renal function in the neonate, elimination of $\mathrm{M} 1$ from the child was prolonged, resulting in substantial increase in exposure of the child to M1. Again the tramadol concentration in the serum samples taken from the child did not deviate from previously reported in a group of neonates. (8) Furthermore, in the study by Claahsen-van der Grinten et al., no relationship between the concentration of tramadol and M1 in the serum of the neonate and APGAR score 1 min post-partum was detected, in concordance with the present case of the child with APGAR score 8/9. (8) At the top of the body system ranking of adverse events in neonates was the respiratory system (14.3\%); the incidence was not related to the tramadol dose.
(8) The possible relationship with the concentration of tramadol or M1 was not commented. However, the authors report the case of respiratory depression in a child probably related to the treatment with the highest concentration of tramadol $(0.557 \mathrm{mg} / \mathrm{l})$ and M1 $(0.112$ $\mathrm{mg} / \mathrm{l})$. The respiratory status in this child immediately improved with naloxone treatment. (8) In our case naloxone was not used, since association of respiratory depression with tramadol use in the mother was not immediately recognized. Our hypothesis that neonatal death could be associated with the UM phenotype of the mother is further supported by a published case report of respiratory depression associated with elevated levels of M1 in an adult patient with renal impairment and CYP2D6 gene duplication who was treated with tramadol. (22) Similar to the situation in newborns, elimination of $\mathrm{M} 1$ is prolonged in patients with renal impairment. To confirm our hypothesis plasma concentration of $\mathrm{M} 1$ should have been measured and CYP2D6 genotype of the mother should have been identified. Nevertheless, in mothers treated for labour pain, tramadol should be administered with more caution until more safety data are available. 


\section{REFERENCES}

1. Gillen C, Haurand M, Kobelt DJ, Wnendt S. Affinity, potency and efficacy of tramadol and its metabolites at the cloned human $\mu$-opioid receptor. Naunyn Schmiedebergs Arch Pharmacol 2000;362:116-21.

2. Raffa RB, Friderichs E, Reimann W, Shank RP, Codd EE, Vaught JL. Opioid and nonopioid components independently contribute to the mechanism of action of tramadol, an 'atypical' opioid analgesic. J Pharmacol Exp Ther 1992;260:275-85.

3. Sachdeva DK, Jolly BT. Tramadol overdose requiring prolonged opioid antagonism. Am J Emerg Med 1997;15:217-8.

4. Vickers MD, O'Flaherty D, Szekely SM, Read M, Yoshizumi J. Tramadol: pain relief by an opioid without depression of respiration. Anaesthesia 1992;47:291-6.

5. Bitsch M, Emmrich J, Hary J, Lippach G, Rindt W. Analgesia in obstetrics with tramadol. Fortschr Med 1980;98:632-4.

6. Viegas OA, Khaw B, Ratnam SS. Tramadol in labour pain in primiparous patients. A prospective comparative clinical trial. Eur J Obstet Gynecol Reprod Biol 1993;49:131-5.

7. Paar WD, Frankus $P$, Dengler HJ. The metabolism of tramadol by human liver microsomes. Clin Investig 1992;70:708-10.

8. Claahsen-van der Grinten HL, Verbruggen I, van den Berg PP, Sporken JMJ, Kollée LAA. Different pharmacokinetics of tramadol in mothers treated for labour pain and in their neonates. Eur J Clin Pharmacol 2005;61:523-9.

9. Allegaert K, Anderson BJ, Verbesselt R, Debeer A, de Hoon J, Devlieger H, et al. Tramadol disposition in the very young: an attempt to assess in vivo cytochrome P-450 $2 \mathrm{D6}$ activity. Br J Anaesth 2005;95:231-9.

10. Keskin HL, Aktepe Keskin E, Avsar AF, Tabuk M, Caglar GS. Pethidine versus tramadol for pain relief during labor. Int J Gynecol Obstet 2003;82:11-6.

11. Khooshideh M, Shahriari A. A comparison of tramadol and pethidine analgesia on the duration of labour: A randomised clinical trial. Aust N Z J Obstet Gynaecol 2009;49:59-63.

12. Marquardt KA, Alsop JA, Albertson TE. Tramadol exposures reported to statewide poison control system. Ann Pharmacother 2005;39:1039-44.

13. Stamer UM, Musshoff F, Kobilay M, Madea B, Hoeft A, Stuber F. Concentrations of tramadol and O-desmethyltramadol enantiomers in different CYP2D6 genotypes. Clin Pharmacol Ther 2007;82:41-7.

14. Gaedigk A, Bradford LD, Marcucci KA, Leeder JS. Unique CYP2D6 activity distribution and genotype-phenotype discordance in black Americans. Clin Pharmacol Ther 2002;72:76-89.

15. Plesnicar BK, Zalar B, Breskvar K, Dolzan V. The influence of the CYP2D6 polymorphism on psychopathological and extrapyramidal symptoms in the patients on long-term antipsychotic treatment. J Psychopharmacol 2006;20:829-33.

16. Sachse C, Brockmoller J, Bauer S, Roots I. Cytochrome P450 2D6 variants in a Caucasian population: allele frequencies and phenotypic consequences. Am J Hum Genet 1997;60:284-95.

17. Willette RN, Doorley BM, Sapru HN. Activation of cholinergic mechanisms in the medulla-oblongata reverse intravenous opioid-induced respiratory depression. J Pharmacol Exp Ther 1987;240:352-8.

18. Naranjo CA, Busto U, Sellers EM, Sandor P, Ruiz I, Roberts EA, et al. A method for estimating the probability of adverse drug reactions. Clin Pharmacol Ther 1981;30:239-45.

19. Lintz W, Barth H, Osterloh G, Schmidt-Bothelt E. Pharmacokinetics of tramadol and bioavailability of enteral tramadol formulations. 3rd Communication: suppositories. Arzneimittelforschung 1998;48:889-99.

20. Garrido M, Habre W, Rombout F, Trocóniz I. Population pharmacokinetic/pharmacodynamic modelling of the analgesic effects of tramadol in pediatrics. Pharm Res 2006;23:2014-23.

21. Poulsen L, Arendt-Nielsen L, Brosen K, Sindrup SH. The hypoalgesic effect of tramadol in relation to CYP2D6. Clin Pharmacol Ther 1996;60:636-44.

22. Stamer UM, Stuber F, Muders T, Musshoff F. Respiratory depression with tramadol in a patient with renal impairment and CYP2D6 gene duplication. Anesth Analg 2008;107:926-9. 